\title{
Construcción de cursos sobre comprensión y producción de textos en educación superior: el caso de la UNAD.
}

Sandra Acevedo Zapata ${ }^{1}$

\begin{abstract}
Resumen
Este artículo presenta el proceso investigativo de carácter descriptivo, a través del cual se diseñó la propuesta de los cursos de comprensión y producción de textos en ambientes virtuales de aprendizaje. En dicho proceso se retomaron las experiencias exitosas y las categorías fundamentales de las investigaciones que demostraron haber aportado más efectivamente en los procesos de escritura y lectura de textos académicos en educación superior.
\end{abstract}

Palabras clave: competencia comunicativa, comprensión de textos, cursos en ambientes virtuales de aprendizaje, producción de textos en educación superior. 


\title{
Construction of classes for comprehension and production of higher education texts: The case of UNAD
}

\begin{abstract}
This study presents a descriptive research process, through which the proposal for courses of comprehension and production of texts in virtual learning environments designed. In this process successful experiences were resumed, as well as the fundamental research categories that have demonstrated contributing most effectively in the processes of writing and reading academic texts in higher education.
\end{abstract}

Key words: Courses in virtual learning environments, reading comprehension, text production in higher education.

\section{Introducción}

En la formación profesional y en el ejercicio de la vida laboral se requiere una excelente capacidad de comprender el mundo y analizarlo para encontrar soluciones a problemas reales que beneficien a la comunidad. En ese orden de ideas, el proceso de formación que brinda la universidad, debe posibilitar una aproximación al mundo desde diferentes perspectivas y con claros criterios que permitan tomar decisiones y desarrollar proyectos exitosamente.

Las Instituciones de Educación Superior (IES) preocupadas por desarrollar los procesos formativos que logren hacer pertinente el perfil profesional y ocupacional de sus estudiantes y egresados, han asumido el reto de formación a partir de la realidad de quienes ingresan como estudiantes desde sus primeros 
semestres. Lo anterior, teniendo en cuenta que se ha identificado que los estudiantes que ingresan a los primeros periodos de educación superior tienen debilidad en los procesos de comprensión lectora y en la escritura de textos académicos, lo que en muchos casos se convierte en una causa de deserción escolar.

Además, en la Universidad Nacional Abierta y a Distancia (UNAD), a la problemática planteada, se suma una exigencia mayor del modelo de educación abierta, a distancia y virtual, el cual implica procesos de autonomía, donde las capacidades de lectura y escritura se vuelven fundamentales. En el mismo sentido, se ha identificado que el $87 \%$ de los estudiantes que recibe la UNAD en el primer periodo, poseen alta vulnerabilidad académica y socioeconómica, lo que ha obligado a crear proyectos de acompañamiento permanente de los estudiantes, con el objetivo de facilitar su estadía y permanencia y de esta manera también disminuir la tasa de deserción.

La UNAD en su Proyecto Académico Solidario Versión 3.0, asume como fundamento misional el componente comunitario que se hace realidad en la interacción social a través de los procesos de comunicación humana, lo que les permite construir identidad como sujeto social en el reconocimiento del otro desde la diversidad como resultado de la interacción en reciprocidad.

En el proceso de formación de los estudiantes se ha diseñado dentro del macro currículo de la UNAD el campo de formación Interdisciplinar Básico Común (IBC) con un componente de Formación en Tecnocultura, Comunicación y Lenguajes, el cual le apunta a la "comprensión de procesos comunicativos en multicontextos y multiformatos que ofrece la posibilidad de construcción de sistemas de lenguaje orientados a la comprensión, análisis y construcción social de significado" (UNAD, 2012:6).

Considerando lo anterior, se requiere que la UNAD asuma una mirada crítica de sus propias prácticas de enseñanza de las competencias comunicativas, que superen el uso de herramientas para profundizar en el sentido de la comunicación, el reconocimiento de las tecnoculturas, los nuevos lenguajes y asuma también la tradición académica a través de la lectura y la escritura en educación superior.

Es en este contexto que se construye una propuesta para los cursos iniciales en el proceso de entrada a la vida universitaria, cuyo objetivo general fue diseñar una propuesta de cursos de comprensión y producción de textos académicos en educación superior que implementen estrategias pedagógicas en ambientes virtuales de aprendizaje. Los objetivos específicos fueron tres. El primero, identificar los hallazgos de las abordajes académicos y las experiencias sobre los procesos de formación en la comprensión lectora de textos académicos en Educación Superior; el segundo, sistematizar y analizar los hallazgos de 
abordajes académicos y las experiencias sobre los procesos de formación en la comprensión lectora de textos académicos en Educación Superior, y el tercero, diseñar los cursos de comprensión y producción de textos y divulgar los resultados de la investigación en ámbitos académicos, además generar un informe, tipo artículo de investigación, sobre la sistematización de la aplicación de la experiencia de los cursos y la divulgación de los hallazgos de la investigación.

\section{Antecedentes}

Las investigaciones en el campo de la lectura y escritura surgieron por la problemática de los estudiantes que llegaban a la educación superior con dificultades de comprensión y construcción de textos que respondiesen a las necesidades de la universidad, lo que se ha vuelto una constante y se evidencia en las recurrentes afirmaciones de los docentes sobre las dificultades que presentan los estudiantes para presentar sus trabajos escritos y de interpretar y apropiar los conceptos de los documentos que se les proponen en los cursos.

Las investigaciones en este ámbito muestran que los estudiantes deben asumir la lectura de textos cada vez más especializados y complejos pero presentan serias dificultades para interpretarlos y dar soporte a los futuros aprendizajes. Por tanto las universidades han asumido este problema y se detectó la necesidad de generar procesos para que los estudiantes aprendan a interpretar textos durante su proceso de formación en la universidad.

Para mejorar los procesos de lectura y escritura de los estudiantes durante la vida académica en la universidad es necesario asumirla desde un abordaje curricular y pedagógico que permita hacer una propuesta pedagógica que intente resolver esta problemática en los primeros periodos. Esto requiere asumir que:

- En el estado del arte de este proceso investigativo se anota que los estudiantes de los primeros periodos de Educación Superior ingresan sin tener el dominio adecuado de procesos de lectura y escritura que les permita abordar exitosamente los textos académicos (Fernández y Carvajal 2002).

- El estudio de los procesos de la comunicación implícitos en la lectura y escritura de textos académicos es fundamental como formación básica de todo profesional.

- Es necesario el manejo adecuado de la lengua desde una perspectiva no solo lingüística sino semiótica, con un adecuado proceso de formación hacia la elección de códigos sociolingüísticos elaborados y universalistas pertinentes a este nivel de formación, lo cual permite ganar elementos y estrategias que facilitan el acceso al conocimiento de las diferentes disciplinas de las profesiones elegidas por el estudiante. 
- Es necesario diseñar cursos que ayuden a los estudiantes a resolver las debilidades que presenten al momento de iniciar su proceso de formación en la universidad. Estas debilidades pueden ser detectadas y evaluadas en una prueba de entrada.

\section{Metodología para la construcción de los cursos}

Se adelantó un proceso investigativo construyendo el estado del arte de las investigaciones en este campo de formación y las experiencias en Educación Superior con cursos relacionados con procesos de lectura y escritura, con estos datos se escribió un artículo de revisión que se ha presentado a publicación en una revista indexada.

La investigación descriptiva, que se desarrolló en este trabajo, abordó las siguientes fases: exploratoria, sistematización y analítica, proyectiva.

Fase exploratoria. Con el propósito de identificar los hallazgos de los abordajes académicos y las experiencias sobre los procesos de formación en la comprensión lectora de textos académicos en educación superior, se realizaron procesos de rastreo, identificación de artículos de revistas fruto de investigaciones o experiencias de universidades nacionales e internacionales en algunos países donde el español es la lengua materna; luego se estableció la tematización y reseña de los mismos.

Fase de sistematización y análisis. Se retomó la información recolectada y se organizaron en matrices de doble entrada por categorías, las cuales generaron hallazgos de abordajes académicos y las experiencias sobre los procesos de formación en la comprensión lectora de textos académicos en educación superior.

Fase proyectiva. Esta fase comprende tres actividades, la primera, el diseño de los cursos iniciales de comprensión y producción de textos en ambientes virtuales de aprendizaje, la segunda, a partir de la experiencia y los resultados obtenidos, dar a conocer el trabajo desarrollado en ámbitos académicos, y la tercera, realizar un informe, tipo artículo de investigación, sobre la sistematización de la aplicación de la experiencia de los cursos que permita divulgar los hallazgos de la investigación.

\section{Discusión de categorías fundamentales}

\section{La evolución de las miradas sobre los procesos de lectura y la escritura}

La discusión fundamental sobre la lectura aporta que leer implica asumir el pensamiento reflexivo, analítico y crítico de un lector intencional, autónomo 
e independiente, es decir como agente activo y responsable de su aprendizaje. Por tanto, se asume un proceso de comprensión centrado en el lector, que aporta al proceso, ya que depende de él la construcción del significado del texto, que a su vez depende de sus esquemas de conocimiento previo, sus expectativas, sus preferencias y sus metas de lectura. Por tanto, la comprensión de lectura se asume como un proceso gradual y estratégico de construcción de sentido, el cual se da a partir de la interacción del lector con el texto en un contexto específico.

Se ha superado la mirada de los años 60 donde un curso de lengua implicaba tan solo enseñar sobre las habilidades lingüísticas: hablar, escuchar, leer y escribir. Donde hablar y escribir se constituían en eventos productivos que demandan un sujeto activo; por su parte, escuchar y leer se consideraban receptivos y configuran un sujeto pasivo.

En los años 70 se hizo evidente que ninguna de las habilidades de la lengua es pasiva. J. Orasen y M. Penney (1986) nos recuerdan que la lectura, en la visión tradicional, se llevaba paso a paso desde la percepción de letras para formar palabras y de su combinación hasta completar oraciones. De esta manera se pasaba, automáticamente, a la comprensión. Se insistía en la práctica de la decodificación y se dedicaba poca atención a la comprensión y a los problemas o dificultades de lectura, los cuales se resolvían con más práctica en la decodificación. Es así como:

- Se llegó a establecer que el significado de las oraciones no es equivalente a la suma de los significados de las palabras.

- La enseñanza a partir de la práctica de repetición como refuerzo no garantizaba la adquisición o el aprendizaje de una lengua.

- Se admitió que en la comprensión y en la producción del discurso el sujeto no sólo reconoce o produce oraciones aisladas, con significado local, sino que además, reconoce su valor y función, o se los asigna, como parte de un todo coherente, del sentido global del texto.

- Se resalta el papel de los esquemas, ya que el conocimiento de los textos, de la manera como se organizan, de sus marcas discursivas conforma esquemas estructurales, formales o textuales; esquemas cognitivos previos esenciales para la construcción del significado del texto. 
La comunicación superó la perspectiva instrumental que describía sus elementos por un abordaje que ahora se centra en el proceso comunicativo sobre el sentido que circula y el significado que se construye en dicha interacción. Perilla (2004) en su investigación sobre el mejoramiento en la comprensión de textos académicos en el ámbito universitario, plantea un abordaje de las ciencias del lenguaje asumiendo la complejidad del mismo que le permite trasformar los objetos de conocimiento y no puede reducirse a la lengua natural porque se reducirían o restringirían aquellos procesos implícitos en la comprensión de textos en la construcción de conocimiento.

\section{Más allá de la sintaxis la búsqueda del significado}

En esta propuesta también se retoman los aportes de Berstein (1985), quien al preguntarse por el problema de las diferencias en el aprendizaje escolar generó la noción de código sociolingüístico, que permite analizar desde la semántica y no solo desde la sintaxis a la producción de textos. Así mismo buscó comprender «la estructuración social de los significados» y sus manifestaciones lingüísticas en contextos diferentes pero relacionados. Un contexto es la institución escolar, en la cual se posibilita el acceso y en sus prácticas se exige el uso de los códigos elaborados propios de los discursos académicos y la claridad de que los estudiantes viven desde la experiencia cotidiana a través de la comunicación donde se privilegia en sus prácticas comunicativas el código restringido que puede limitar los referentes y las estructuras simbólicas, por tanto se requiere que el sujeto protagonista del proceso de aprendizaje pueda utilizar los códigos sociolingüísticos en su sentido más amplio sobre la comprensión de los discursos generando posturas críticas profundas y propositivas para el diseño de soluciones y la trasformación para su vida y de la sociedad.

\section{Lectura como proceso semiótico del texto de los procesos inherentes a su riqueza en posibilidades de construcción de sentido}

Los profesionales de todos los campos realizan habitualmente la lectura y construcción de textos en sus diferentes géneros, los cuales son relacionados con el discurso de acuerdo a prácticas sociales donde se construyen y a las cuales corresponden.

El texto es una noción que según Eco (1979: 73) «artificio sintáctico, semántico y pragmático» es una cadena de artificios expresivos que el destinatario debe actualizar, pues el texto requiere ser completado en lo que no se ha dicho. 


\section{La escritura como producción de sentido}

La semiótica es una teoría de significación basada en enunciación desde un sistema de representación que va construyendo el sujeto y que él requiere para comprender los conocimientos teóricos y prácticos a través de diversos modos de representación. La comunicación implica la enunciación, el compartir el código y principalmente la resemiotización del enunciado por parte del destinatario.

Retomando las conclusiones de María Ángeles Echevarría (2006) “...La comprensión implica un conglomerado de procesos, y que dependiendo de que todos o solo algunos se pongan en funcionamiento, podemos hablar de una comprensión más o menos profunda o superficial e, incluso, de una lectura no comprensiva". Para obtener una comprensión completa o profunda es necesario que el sujeto culminé con éxito los siguientes procesos:

1. Extraer las ideas de las palabras y oraciones del texto (generar una microestructura).

2. Realizar las inferencias que garanticen las relaciones de coherencia entre las ideas de modo que se pueda componer un hilo conductor entre ellas.

3. Suprimir, generalizar e integrar la información que proporciona el texto y construir las ideas globales que contienen la información más importante y que le confieren unidad (generar macroestructura).

4. Reconocer la trama de relaciones que articulan las ideas globales (la superestructura).

5. Elaborar una representación propia de lo que el texto comunica.

Los problemas que evidencian los sujetos de la investigación antes de la intervención nos llevan a pensar que no culminan con éxito los tres últimos procesos y, por tanto, no alcanzan los niveles de profundidad esperables en este tipo de estudiantes, (Echevarría, 2006:186). 


\section{Estructura curricular de los cursos de comprensión y producción de textos}

\section{-Núcleo problémico}

¿Cómo mejorar los procesos de lectura y escritura para comprender de manera significativa y crítica el mundo con los referentes que aporta la academia a partir de la educación superior?

\section{-Microcurrículos en ambientes virtuales de aprendizaje}

\begin{tabular}{|c|c|c|}
\hline & \multicolumn{2}{|c|}{ Microcurrículos en AVA } \\
\hline $\begin{array}{c}\text { Se sugiere realizar una } \\
\text { Evaluación Diagnóstica }\end{array}$ & \multicolumn{2}{|c|}{ Momento sugerido para tomar los curso } \\
\hline Cursos & $\begin{array}{c}\text { Primalizando formación } \\
\text { Comprensión de textos } \\
\text { académicos } \\
(3 \text { créditos })\end{array}$ & $\begin{array}{c}\text { Fisciplinar de grado o iniciando } \\
\text { formación de posgrado }\end{array}$ \\
\hline & $\begin{array}{c}\text { Estrategias de lectura y análisis } \\
\text { de textos científicos (3 créditos) }\end{array}$ \\
\hline & Escritura de textos académicos \\
$(3$ créditos $)$ & $\begin{array}{c}\text { Escritura de artículos científicos } \\
\text { (3 créditos })\end{array}$ \\
\hline
\end{tabular}

\section{-Ruta curricular}

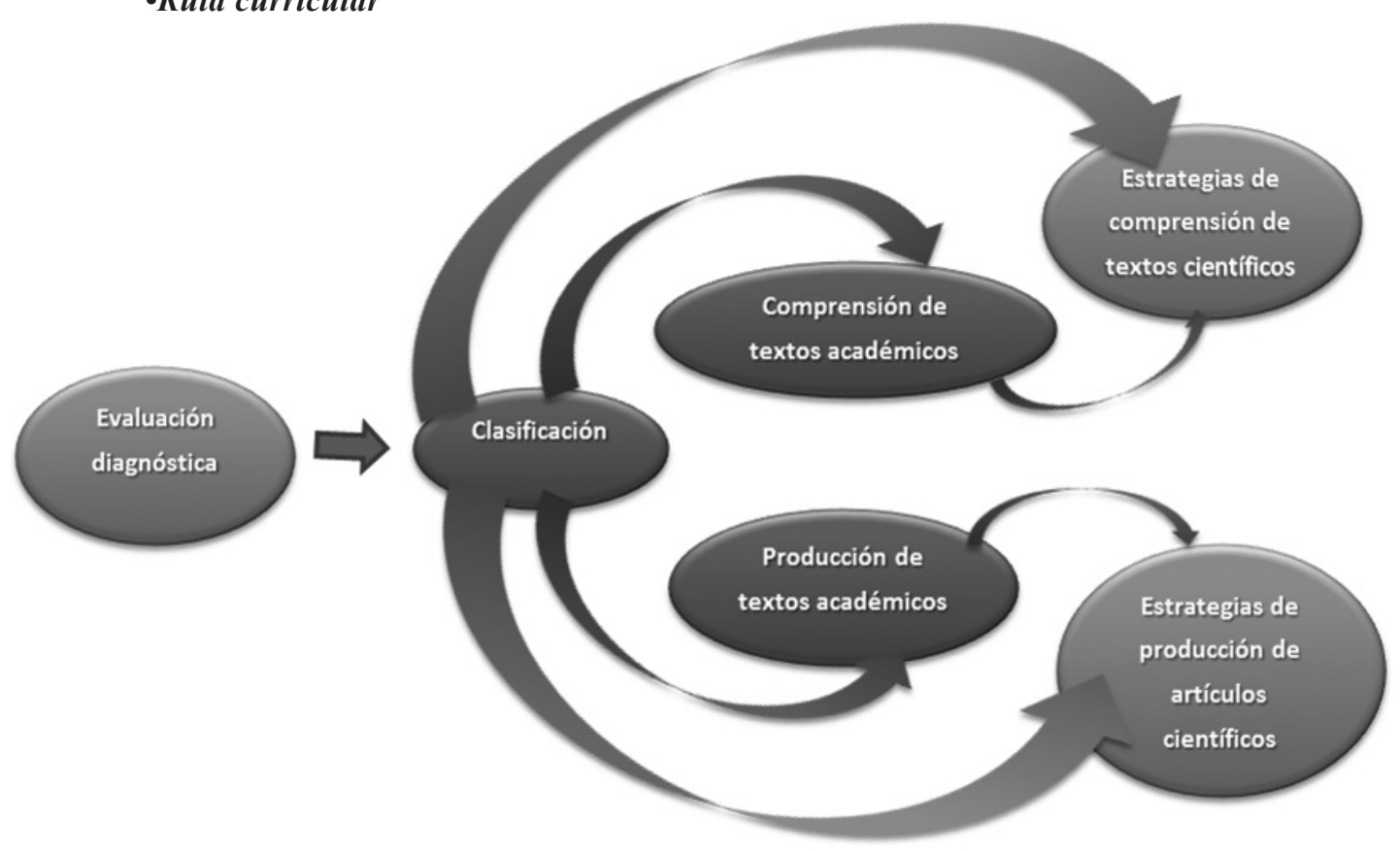




\section{Estrategias pedagógicas y evaluativas en Ambientes Virtuales de Aprendizaje (AVA)}

Los Ambientes Virtuales de Aprendizaje en la UNAD se diseñaron siguiendo la disposición de elementos que permite o propicia el autoaprendizaje y son coherentes con el actual proceso de innovación curricular. Las estrategias pedagógicas y estrategias evaluativas propuestas retoman la experiencia de enseñanza de los docentes de diferentes universidades tanto en sus cursos como en sus investigaciones y especialmente la del grupo de la Universidad Central participante, donde ya se dio este proceso de virtualización de estos cursos y se generó un buen resultado.

\section{Elementos constitutivos de los cursos de comprensión y producción de textos}

\begin{tabular}{|c|c|}
\hline CURSO & DESCRIPCIÓN \\
\hline $\begin{array}{l}\text { Competencias comunicativas } \\
\text { implícitas en la comprensión y } \\
\text { producción de textos }\end{array}$ & $\begin{array}{l}\text { Comprensión lectora: } \\
\text {-Abordar la comprensión a través de ejercicios representativos que } \\
\text { propicien el adecuado manejo de las diferentes estrategias que } \\
\text { permitan la apropiación de la estructura de los textos académicos } \\
\text { en educación superior a través de estrategias de comprensión. } \\
\text { Producción Textual: } \\
\text { - Conocer el proceso de producción textual en el ámbito académico } \\
\text { de la educación superior: Formas de expresión escrita. La } \\
\text { descripción. La narración. La argumentación. Estructura de textos } \\
\text { argumentativos. Tipos de argumentos. La exposición. Textos } \\
\text { Académicos: El ensayo. Tipos de ensayo. El informe científico. } \\
\text { Textos científicos. } \\
\text { Semiótica de la imagen: } \\
\text {-Brindar elementos críticos para que los estudiantes puedan analizar } \\
\text { las propuestas icónicas, artísticas, que se presentan a través de los } \\
\text { medios y las redes de comunicación que se han potenciado con el } \\
\text { uso de la internet. } \\
\text { Comunicación digital: } \\
\text {-Diseñar propuestas comunicativas que permitan producir nuevos } \\
\text { mensajes y significados con los recursos multimediales y que } \\
\text { utilizan los diferentes medios como el video, multimedia, hipermedia } \\
\text { en los multicontextos. }\end{array}$ \\
\hline
\end{tabular}

\section{Conclusiones}

A continuación, y teniendo en cuenta las diferentes actividades desarrolladas en este trabajo, se presentan las conclusiones, las cuales están organizadas considerando los dos procesos: lectura y escritura. 


\section{Con respecto a los procesos de comprensión de textos en educación virtual:}

-El curso virtual de procesos de comprensión y producción de textos académicos, surge de la necesidad de optimizar los procesos de lectura y escritura del estudiante en la práctica académica universitaria, para propiciar la construcción del conocimiento.

-De ahí que dicho espacio se fundamente bajo de tres pilares teóricos: el aprendizaje significativo-colaborativo, la meta-cognición y la lingüística textual.

-Para el desarrollo de un curso de comprensión de textos en ambientes virtuales este espacio y a distancia, como demandante de formas diferentes de lectura que depende del modo de acercamiento al conocimiento en el que se quiere hacer énfasis.

\section{Con respecto a los proceso de escritura en educación virtual:}

- La escritura es un lenguaje para comunicar ideas por medio de signos convencionales y visibles que traspasan el tiempo y el espacio.

- Como sistema de comunicación se debe aprender, por ello, requiere desarrollar habilidades propias de la producción de sentido y significado en la escritura para poder comunicarse.

- Desde esta perspectiva, la escritura reestructura la conciencia, como dice Ong (1987), porque es un proceso propio del pensamiento y a través de ella es posible reconocernos.

- Escribir en la universidad implica pasar de la opinión inmediata al proceso cognitivo de razonamiento y reflexión para reconocer conceptos y problematizarlos por medio de razones o planteamientos que sustenten su veracidad y se llegue a la construcción de conocimiento.

-Por tanto, se debe llegar a desarrollar estructuras explicativas y argumentativas dentro del discurso escrito para asegurar una interpretación clara por medio de un proceso en el estudiante de pensamiento deductivo, inductivo, abductivo y analítico.

-Es así como, la construcción de un escrito, también es un proceso que debe pasar por unos momentos para llegar a un resultado donde exista la armonía entre el manejo del código y la competencia textual e intelectual que posee el autor. 
-Por lo anterior, en el ámbito de la escritura académica es requerido como competencia comunicativa la escritura del texto argumentativo, vista desde una lógica de la composición.

\section{Referencias bibliográficas}

Ausubel, D. 1983. Psicología Educativa: Un punto de vista cognoscitivo. México: Trillas.

Berstein, B. 1985. Clases sociales, lenguaje y socialización. Revista colombiana de educación.15:18_34

Bruner, J. 1991. Actos de significado: más allá de la revolución cognitiva. Madrid: Alianza.

Bruner, J. 1990. La elaboración del sentido: la construcción del mundo por el niño. Barcelona: Paidós.

Eco, U. 1998. Signo. Barcelona: Labor.

Eco, U. 1979. Lector in fábula. La cooperación interpretativa en el texto narrativo. Barcelona: Lumen.

Echevarría, M.A. 2006. ¿Enseñar a leer en la universidad? Una intervención para mejorar la comprensión de textos complejos al comienzo de la educación superior. Revista de psicodidáctica. 11(2): 169-188.

Escudero, G. y Santana, B. 1985. Los modelos del proceso de la escritura. Revista Estudios de Psicología, 19-20: 87-101.

Fernández, M.T. y Carvajal, M. 2002. Niveles de alfabetización en educación superior. México: Universidad Autónoma de Aguas Calientes.

Ferreiro E. 1999. Cultura, escrita y educación. México: Fondo de cultura económica.

González, S. 2010. Modulo de lectura. Bogotá: Universidad Central.

González, S. 2007. Una experiencia en ambientes educativos virtuales para el desarrollo de la lectura. Pedagogía y saberes. Bogotá: Universidad Pedagógica Nacional.

Nóbile, N. 2008. Escritura electrónica y nuevas formas de subjetividad. Blog la citrola del sociólogo. En http://superintelectual.blogspot.com/2008/07/escritura-electrnicay-nuevas-formas-de.html

Ong, W. 1987. Oralidad y escritura: tecnologías de la palabra. Traducción de Angélica Scherp, México: Fondo de Cultura Económica. 


\section{Revista de}

investigaciones UNAD

Volumen 12. Número 2. Diciembre 2013

Perilla, A., Rincón, G., Gil, J.S. Salas, R. 2004. El mejoramiento de los procesos de comprensión de textos académicos en el ámbito universitario. Revista Lenguaje, 32: 159-182.

Sánchez, J. 2007. Saber escribir. Bogotá: Editorial Aguilar.

UNAD, Comité Asesor de Currículo. 2012, Ruta para el diseño de programas articulados en torno a problemas y competencias. VIACI- UNAD.

Van Dijk, T. A. (Comp.). 2000. El discurso como interacción social. Estudios sobre el discurso II. Una introducción multidisciplinaria. Barcelona: Gedisa. 